Revised version

June 2003

\title{
Reviewing the problem of the $U(1)$ axial symmetry and the chiral transition in QCD
}

\author{
M. Marchi, E. Meggiolaro \\ Dipartimento di Fisica, \\ Università di Pisa, \\ Via Buonarroti 2, \\ I-56127 Pisa, Italy.
}

\begin{abstract}
We discuss the role of the $U(1)$ axial symmetry for the phase structure of QCD at finite temperature. We expect that, above a certain critical temperature, also the $U(1)$ axial symmetry will be (effectively) restored. We will try to see if this transition has (or has not) anything to do with the usual chiral transition: various possible scenarios are discussed. In particular, supported by recent lattice results, we analyse a scenario in which a $U(1)-$ breaking condensate survives across the chiral transition. This scenario can be consistently reproduced using an effective Lagrangian model. The effects of the $U(1)$ chiral condensate on the slope of the topological susceptibility in the full theory with quarks are studied: we find that this quantity (in the chiral limit of zero quark masses) acts as an order parameter for the $U(1)$ axial symmetry above the chiral transition. Further information on the new $U(1)$ chiral order parameter is derived from the study (at zero temperature) of the radiative decays of the "light" pseudoscalar mesons in two photons: a comparison of our results with the experimental data is performed.
\end{abstract}

(PACS codes: 12.38.Aw, 12.39.Fe, 11.15.Pg, 11.30.Rd) 


\section{Introduction}

It is generally believed that a phase transition which occurs in QCD at a finite temperature is the restoration of the spontaneously broken $S U(L) \otimes S U(L)$ chiral symmetry in association with $L$ massless quarks. At zero temperature the chiral symmetry is broken spontaneously by the condensation of $q \bar{q}$ pairs and the $L^{2}-1 J^{P}=0^{-}$mesons are just the Goldstone bosons associated with this breaking [1]. At high temperatures the thermal energy breaks up the $q \bar{q}$ condensate, leading to the restoration of chiral symmetry. We expect that this property not only holds for massless quarks but also continues for a small mass region. The order parameter for the chiral symmetry breaking is apparently $\langle\bar{q} q\rangle \equiv \sum_{i=1}^{L}\left\langle\bar{q}_{i} q_{i}\right\rangle$ : the chiral symmetry breaking corresponds to the non-vanishing of $\langle\bar{q} q\rangle$ in the chiral limit $\sup \left(m_{i}\right) \rightarrow 0$. From lattice determinations of the chiral order parameter $\langle\bar{q} q\rangle$ one knows that the $S U(L) \otimes S U(L)$ chiral phase transition temperature $T_{c h}$, defined as the temperature at which the chiral condensate $\langle\bar{q} q\rangle$ goes to zero (in the chiral limit $\sup \left(m_{i}\right) \rightarrow 0$ ), is nearly equal to the deconfining temperature $T_{c}$ (see, e.g., Ref. [2]). But this is not the whole story: QCD possesses not only an approximate $S U(L) \otimes S U(L)$ chiral symmetry, for $L$ light quark flavours, but also a $U(1)$ axial symmetry (at least at the classical level) [3, 4]. The role of the $U(1)$ symmetry for the finite temperature phase structure has been so far not well studied and it is still an open question of hadronic physics whether the fate of the $U(1)$ chiral symmetry of QCD has or has not something to do with the fate of the $S U(L) \otimes S U(L)$ chiral symmetry.

In the "Witten-Veneziano mechanism" [5, 6, for the resolution of the $U(1)$ problem, a fundamental role is played by the so-called "topological susceptibility" in a QCD without quarks, i.e., in a pure Yang-Mills (YM) theory, in the large- $N_{c}$ limit $\left(N_{c}\right.$ being the number of colours):

$$
A=\lim _{k \rightarrow 0} \lim _{N_{c} \rightarrow \infty}\left\{-i \int d^{4} x e^{i k x}\langle T Q(x) Q(0)\rangle\right\},
$$

where $Q(x)=\frac{g^{2}}{64 \pi^{2}} \varepsilon^{\mu \nu \rho \sigma} F_{\mu \nu}^{a} F_{\rho \sigma}^{a}$ is the so-called "topological charge density". This quantity enters into the expression for the mass of the $\eta^{\prime}$. Therefore, in order to study the role of the $U(1)$ axial symmetry for the full theory at non-zero temperatures, one should consider the YM topological susceptibility $A(T)$ at a given temperature $T$, formally defined as in Eq. (1.1), where now $\langle\ldots\rangle$ stands for the expectation value in the full theory at the temperature $T$ [7]. 
The problem of studying the behaviour of $A(T)$ as a function of the temperature $T$ was first addressed, in lattice QCD, in Refs. [8, 9, 10]. Recent lattice results [11] (obtained for the $S U(3)$ pure-gauge theory) show that the YM topological susceptibility $A(T)$ is approximately constant up to the critical temperature $T_{c} \simeq T_{c h}$, it has a sharp decrease above the transition, but it remains different from zero up to $\sim 1.2 T_{c}$. We recall that, in the Witten-Veneziano mechanism [5, 6, a (no matter how small!) value different from zero for $A$ is related to the breaking of the $U(1)$ axial symmetry, since it implies the existence of a would-be Goldstone particle with the same quantum numbers of the $\eta^{\prime}$ (see also Ref. [12]).

Another way to address the same question is to look at the behaviour at non-zero temperatures of the susceptibilities related to the propagators for the following meson channels [13] (we consider for simplicity the case of $L=2$ light flavours): the isoscalar $I=0$ scalar channel $\sigma$ (also known as $f_{0}$ in the modern language of hadron spectroscopy), interpolated by the operator $O_{\sigma}=\bar{q} q$; the isovector $I=1$ scalar channel $\delta$ (also known as $a_{0}$ ), interpolated by the operator $\vec{O}_{\delta}=\bar{q} \frac{\vec{\tau}}{2} q$; the isovector $I=1$ pseudoscalar channel $\pi$, interpolated by the operator $\vec{O}_{\pi}=i \bar{q} \gamma_{5} \frac{\vec{\tau}}{2} q$; the isoscalar $I=0$ pseudoscalar channel $\eta^{\prime}$, interpolated by the operator $O_{\eta^{\prime}}=i \bar{q} \gamma_{5} q$. Under $S U(2)_{A}$ transformations, $\sigma$ is mixed with $\pi$ : thus the restoration of this symmetry at $T_{c h}$ requires identical correlators for these two channels. Another $S U(2)$ chiral multiplet is $\left(\delta, \eta^{\prime}\right)$. On the contrary, under the $U(1)_{A}$ transformations, $\pi$ is mixed with $\delta$ : so, an "effective restoration" of the $U(1)$ axial symmetry should imply that these two channels become degenerate, with identical correlators. Another $U(1)$ chiral multiplet is $\left(\sigma, \eta^{\prime}\right)$. (Clearly, if both chiral symmetries are restored, then all $\pi, \eta^{\prime}, \sigma$ and $\delta$ correlators should become the same.) In practice, one can construct, for each meson channel $f$, the corresponding chiral susceptibility

$$
\chi_{f}=\int d^{4} x\left\langle O_{f}(x) O_{f}^{\dagger}(0)\right\rangle
$$

and then define two order parameters: $\chi_{S U(2) \otimes S U(2)} \equiv \chi_{\sigma}-\chi_{\pi}$, and $\chi_{U(1)} \equiv \chi_{\delta}-\chi_{\pi}$. If an order parameter is non-zero in the chiral limit, then the corresponding symmetry is broken. Present lattice data for these quantities seem to indicate that the $U(1)$ order parameter survives across $T_{c h}$, up to $\sim 1.2 T_{c h}$, where the $\delta-\pi$ splitting is small but still different from zero [14, 15, 16]. In terms of the left-handed and right-handed quark fields, ${ }^{*}$ one has the following expression for the difference between the correlators for the

\footnotetext{
${ }^{*}$ Throughout this paper we use the following notations: $q_{L, R} \equiv \frac{1}{2}\left(1 \pm \gamma_{5}\right) q$, with $\gamma_{5} \equiv-i \gamma^{0} \gamma^{1} \gamma^{2} \gamma^{3}$.
} 
$\delta^{+}$and $\pi^{+}$channels:

$$
\begin{aligned}
& \mathcal{D}_{U(1)}(x) \equiv\left\langle O_{\delta^{+}}(x) O_{\delta^{+}}^{\dagger}(0)\right\rangle-\left\langle O_{\pi^{+}}(x) O_{\pi^{+}}^{\dagger}(0)\right\rangle \\
& \quad=2\left[\left\langle\bar{u}_{R} d_{L}(x) \cdot \bar{d}_{R} u_{L}(0)\right\rangle+\left\langle\bar{u}_{L} d_{R}(x) \cdot \bar{d}_{L} u_{R}(0)\right\rangle\right] .
\end{aligned}
$$

(The integral of this quantity, $\int d^{4} x \mathcal{D}_{U(1)}(x)$, is just equal to the $U(1)$ chiral susceptibility $\left.\chi_{U(1)}=\chi_{\delta}-\chi_{\pi}.\right)$ What happens below and above $T_{c h}$ ? Below $T_{c h}$, in the chiral limit $\sup \left(m_{i}\right) \rightarrow 0$, the left-handed and right-handed components of a given light quark flavour ( up or down, in our case with $L=2$ ) can be connected through the $q \bar{q}$ chiral condensate, giving rise to a non-zero contribution to the quantity $\mathcal{D}_{U(1)}(x)$ in Eq. (1.3) (i.e., to the quantity $\left.\chi_{U(1)}\right)$. But above $T_{c h}$ the $q \bar{q}$ chiral condensate is zero: so, how can the quantity $\mathcal{D}_{U(1)}(x)$ (i.e., the quantity $\left.\chi_{U(1)}\right)$ be different from zero also above $T_{c h}$, as indicated by present lattice data? The only possibility in order to solve this puzzle seems to be that of requiring the existence of a genuine four-fermion local condensate, which is an order parameter for the $U(1)$ axial symmetry and which remains different from zero also above $T_{c h}$. This new condensate will be discussed in Section 3. The rest of the paper will be essentially devoted to the analysis of some interesting phenomenological consequences deriving from this hypothesis.

The paper is organized as follows. In Section 2 we discuss the role of the $U(1)$ axial symmetry for the phase structure of QCD at finite temperature. One expects that, above a certain critical temperature, also the $U(1)$ axial symmetry will be (effectively) restored. We will try to see if this transition has (or has not) anything to do with the usual chiral transition: various possible scenarios are discussed. In particular, supported by the above-mentioned lattice results, in Sections 3 and 4 we analyse a scenario in which a new $U(1)$-breaking condensate survives across the chiral transition and it is still present above $T_{c h}$. This scenario can be consistently reproduced using an effective Lagrangian model, which also includes the new $U(1)$ chiral condensate. This theoretical model was originally proposed in Refs. [17, 18, 19] and is summarized in Sections 3 and 4 for the convenience of the reader. (See also Refs. [20, 21] for a recent review on these problems.) In Section 5 (which, together with Section 6, contains the main original results of this paper) we analyse the consequences of our theoretical model on the slope of the topological susceptibility $\chi^{\prime}$, in the full theory with quarks, showing how this quantity is modified by the presence of a new $U(1)$ chiral order parameter: we will find that $\chi^{\prime}$ (in the chiral limit $\left.\sup \left(m_{i}\right) \rightarrow 0\right)$ acts as an order parameter for the $U(1)$ axial symmetry above $T_{c h}$. Further information on the new $U(1)$ chiral order parameter is derived in Section 6 from the study 
(at zero temperature) of the radiative decays of the "light" pseudoscalar mesons in two photons: a comparison of our results with the experimental data is performed. Finally, the conclusions and an outlook are given in Section 7.

\section{The phase transitions of QCD}

One expects that, above a certain critical temperature, also the $U(1)$ axial symmetry will be (effectively) restored.* We will try to see if this transition has (or has not) anything to do with the usual chiral transition. Let us define the following temperatures:

- $T_{c h}$ : the temperature at which the chiral condensate $\langle\bar{q} q\rangle$ goes to zero. The chiral symmetry $S U(L) \otimes S U(L)$ is spontaneously broken below $T_{c h}$ and it is restored above $T_{c h}$.

- $T_{\chi}$ : the temperature at which the pure-gauge topological susceptibility $A$ drops to zero. Present lattice results indicate that $T_{\chi} \geq T_{c h}[11]$.

- $T_{U(1)}$ : the temperature at which the $U(1)$ axial symmetry is (effectively) restored, meaning that, for $T>T_{U(1)}$, there are no $U(1)$-breaking condensates. If $\langle\bar{q} q\rangle \neq$ 0 also the $U(1)$ axial symmetry is broken, i.e., the chiral condensate is an order parameter also for the $U(1)$ axial symmetry. Therefore we must have: $T_{U(1)} \geq T_{c h}$. Moreover, the Witten-Veneziano mechanism implies that $T_{U(1)} \geq T_{\chi}$, since, after all, the pure-YM topological susceptibility $A$ is a $U(1)$-breaking condensate.

The following scenario, that we will call "SCENARIO 1", in which $T_{\chi}<T_{c h}$, is, therefore, immediately ruled out. In this case, in the range of temperatures between $T_{\chi}$ and $T_{c h}$ the anomaly effects are absent, but the $U(1)$ axial symmetry is still broken by the chiral condensate. In other words, in this range of temperatures the $U(1)$ axial symmetry is spontaneously broken ( $\grave{a}$ la Goldstone) and the $\eta^{\prime}$ is the corresponding Goldstone boson, i.e., it is massless in the chiral limit $\sup \left(m_{i}\right) \rightarrow 0$, or, at least, as light as the pion $\pi$,

* Of course, the $U(1)$ axial symmetry is explicitly broken by the anomaly, which never disappears: the precise meaning of this "effective restoration" will be explained below. 
when including the quark masses. This scenario was first discussed (and indeed really supported!) in Ref. [22].

Therefore, we are left essentially with the two following scenarios.

SCENARIO 2: $T_{c h} \leq T_{U(1)}$, with $T_{c h} \sim T_{\chi} \sim T_{U(1)}$. If $T_{c h}=T_{\chi}=T_{U(1)}$, then, in the case of $L=2$ light flavours, the restored symmetry across the transition is $U(1)_{A} \otimes S U(2)_{L} \otimes$ $S U(2)_{R} \sim O(2) \otimes O(4)$, which may yield a first-order phase transition (see, for example, Ref. [23]).

SCENARIO 3: $T_{c h} \ll T_{U(1)}$, that is, the complete $U(L)_{L} \otimes U(L)_{R}$ chiral symmetry is restored only well inside the quark-gluon plasma domain. In the case of $L=2$ light flavours, we then have at $T=T_{c h}$ the restoration of $S U(2)_{L} \otimes S U(2)_{R} \sim O(4)$. Therefore, we can have a second-order phase transition with the $O(4)$ critical exponents. $L=2$ QCD at $T \simeq T_{c h}$ and the $O(4)$ spin system should belong to the same universality class. An effective Lagrangian describing the softest modes is essentially the Gell-Mann-Levy linear sigma model, the same as for the $O(4)$ spin systems (see Ref. [22]). If this scenario is true, one should find the $O(4)$ critical indices for the $q \bar{q}$ chiral condensate and the specific heat: $\langle\bar{q} q\rangle \sim\left|\left(T-T_{c h}\right) / T_{c h}\right|^{0.38 \pm 0.01}$, and $C(T) \sim\left|\left(T-T_{c h}\right) / T_{c h}\right|^{0.19 \pm 0.06}$. Present lattice data partially support these results.

\section{The $U(1)$ chiral order parameter}

We make the assumption (discussed in the previous sections) that the breaking/restoration of the $U(1)$ chiral symmetry is completely independent of the $S U(L) \otimes S U(L)$ symmetry. The usual chiral order parameter $\langle\bar{q} q\rangle$ is an order parameter both for $S U(L) \otimes S U(L)$ and for $U(1)_{A}$ : when it is different from zero, $S U(L) \otimes S U(L)$ is broken down to $S U(L)_{V}$ and also $U(1)_{A}$ is broken. Thus we need another quantity which could be an order parameter only for the $U(1)$ chiral symmetry [17, 18, 19, 24]. The most simple quantity of this kind was found by 'tHooft in Ref. [4. For a theory with $L$ light quark flavours, it is a $2 L$-fermion interaction that has the chiral transformation properties of:

$$
\mathcal{L}_{e f f} \sim \operatorname{det}_{s t}\left(\bar{q}_{s R} q_{t L}\right)+\operatorname{det}_{s t}\left(\bar{q}_{s L} q_{t R}\right)
$$

where $s, t=1, \ldots, L$ are flavour indices, but the colour indices are arranged in a more general way (see Refs. [19, 24]). It is easy to verify that $\mathcal{L}_{\text {eff }}$ is invariant under $S U(L) \otimes$ 
$S U(L) \otimes U(1)_{V}$, while it is not invariant under $U(1)_{A}$. To obtain an order parameter for the $U(1)$ chiral symmetry, one can simply take the vacuum expectation value of $\mathcal{L}_{\text {eff }}$ : $C_{U(1)}=\left\langle\mathcal{L}_{e f f}\right\rangle$. The arbitrarity in the arrangement of the colour indices can be removed if we require that the new $U(1)$ chiral condensate is "independent" of the usual chiral condensate $\langle\bar{q} q\rangle$, as explained in Refs. [19, 24]. In other words, the condensate $C_{U(1)}$ is chosen to be a genuine $2 L$-fermion condensate, with a zero "disconnected part", the latter being the contribution proportional to $\langle\bar{q} q\rangle^{L}$, corresponding to retaining the vacuum intermediate state in all the channels and neglecting the contributions of all the other states. As a remark, we observe that the condensate $C_{U(1)}$ so defined turns out to be of order $\mathcal{O}\left(g^{2 L-2} N_{c}^{L}\right)=\mathcal{O}\left(N_{c}\right)$ in the large $-N_{c}$ expansion, exactly as the chiral condensate $\langle\bar{q} q\rangle$.

The existence of a new $U(1)$ chiral order parameter has of course interesting physical consequences, which can be revealed by analysing some relevant QCD Ward Identities (WI's) (see Ref. [18] and also Ref. [20]). In the case of the $S U(L) \otimes S U(L)$ chiral symmetry, one immediately derives the following WI:

$$
\int d^{4} x\left\langle T \partial^{\mu} A_{\mu}^{a}(x) i \bar{q} \gamma_{5} T^{b} q(0)\right\rangle=i \delta_{a b} \frac{1}{L}\langle\bar{q} q\rangle
$$

where $A_{\mu}^{a}=\bar{q} \gamma_{\mu} \gamma_{5} T^{a} q$ are the $S U(L)$ axial currents. If $\langle\bar{q} q\rangle \neq 0$ (in the chiral limit $\sup \left(m_{i}\right) \rightarrow 0$ ), the anomaly-free WI (3.2) implies the existence of $L^{2}-1$ non-singlet Goldstone bosons, interpolated by the hermitian fields $O_{b}=i \bar{q} \gamma_{5} T^{b} q$. Similarly, in the case of the $U(1)$ axial symmetry, one finds that:

$$
\int d^{4} x\left\langle T \partial^{\mu} J_{5, \mu}(x) i \bar{q} \gamma_{5} q(0)\right\rangle=2 i\langle\bar{q} q\rangle
$$

where $J_{5, \mu}=\bar{q} \gamma_{\mu} \gamma_{5} q$ is the $U(1)$ axial current. But this is not the whole story! One also derives the following WI:

$$
\int d^{4} x\left\langle T \partial^{\mu} J_{5, \mu}(x) O_{P}(0)\right\rangle=2 \operatorname{Li}\left\langle\mathcal{L}_{e f f}(0)\right\rangle
$$

where $\mathcal{L}_{\text {eff }}$ is the $2 L$-fermion operator defined by Eq. (3.1), while the hermitian field $O_{P}$ is defined as: $O_{P} \sim i\left[\operatorname{det}\left(\bar{q}_{s R} q_{t L}\right)-\operatorname{det}\left(\bar{q}_{s L} q_{t R}\right)\right]$. If the $U(1)$-breaking condensate survives across the chiral transition at $T_{c h}$, i.e., $C_{U(1)}=\left\langle\mathcal{L}_{\text {eff }}(0)\right\rangle \neq 0$ for $T>T_{c h}$ (while $\langle\bar{q} q\rangle=0$ 
for $T>T_{c h}$ ), then this WI implies the existence of a (would-be) Goldstone boson (in the large $-N_{c}$ limit) coming from this breaking and interpolated by the hermitian field $O_{P}$. Therefore, the $U(1)_{A}\left(\right.$ would-be) Goldstone boson (i.e., the $\left.\eta^{\prime}\right)$ is an "exotic" $2 L$-fermion state for $T>T_{c h}$.

\section{The new chiral effective Lagrangian}

The proposed scenario, in which the $U(1)$ axial symmetry is (effectively) restored at a temperature $T_{U(1)}$ greater than $T_{c h}$, can be consistently reproduced using an effectiveLagrangian model. This analysis was originally performed in Refs. [17, 18, 19] and is summarized here for the convenience of the reader.

It is well known that the low-energy dynamics of the pseudoscalar mesons, including the effects due to the anomaly and the $q \bar{q}$ chiral condensate, and expanding to the first order in the light quark masses, can be described, in the large- $N_{c}$ limit, by an effective Lagrangian [25, 26, 27, 28, 29] written in terms of the mesonic field $U_{i j} \sim \bar{q}_{j R} q_{i L}$ (up to a multiplicative constant) and the topological charge density $Q$. We make the assumption that there is a $U(1)$-breaking condensate which stays different from zero across $T_{c h}$, up to $T_{U(1)}>T_{c h}$ : the form of this condensate has been discussed in the previous section. We must now define a field variable $X$, associated with this new condensate, to be inserted in the chiral Lagrangian. The translation from the fundamental quark fields to the effectiveLagrangian meson fields is done as follows. The operators $i \bar{q} \gamma_{5} q$ and $\bar{q} q$ entering in the WI (3.3) are essentially equal to (up to a multiplicative constant) $i\left(\operatorname{Tr} U-\operatorname{Tr} U^{\dagger}\right.$ ) and $\operatorname{Tr} U+\operatorname{Tr} U^{\dagger}$ respectively. Similarly, the operators $\mathcal{L}_{\text {eff }} \sim \operatorname{det}\left(\bar{q}_{s R} q_{t L}\right)+\operatorname{det}\left(\bar{q}_{s L} q_{t R}\right)$ and $O_{P} \sim i\left[\operatorname{det}\left(\bar{q}_{s R} q_{t L}\right)-\operatorname{det}\left(\bar{q}_{s L} q_{t R}\right)\right]$ entering in the WI (3.4) can be put equal to (up to a multiplicative constant) $X+X^{\dagger}$ and $i\left(X-X^{\dagger}\right)$ respectively, where $X \sim \operatorname{det}\left(\bar{q}_{s R} q_{t L}\right)$ is the new field variable (up to a multiplicative constant), related to the new $U(1)$ chiral condensate, which must be inserted in the chiral effective Lagrangian. It was shown in Refs. [17, 18, 19] that the most simple effective Lagrangian, constructed with the fields $U, X$ and $Q$, is:

$$
\mathcal{L}\left(U, U^{\dagger}, X, X^{\dagger}, Q\right)=\frac{1}{2} \operatorname{Tr}\left(\partial_{\mu} U \partial^{\mu} U^{\dagger}\right)+\frac{1}{2} \partial_{\mu} X \partial^{\mu} X^{\dagger}
$$




$$
\begin{aligned}
& -V\left(U, U^{\dagger}, X, X^{\dagger}\right)+\frac{1}{2} i Q(x) \omega_{1} \operatorname{Tr}\left(\ln U-\ln U^{\dagger}\right) \\
& +\frac{1}{2} i Q(x)\left(1-\omega_{1}\right)\left(\ln X-\ln X^{\dagger}\right)+\frac{1}{2 A} Q^{2}(x),
\end{aligned}
$$

where the potential term $V\left(U, U^{\dagger}, X, X^{\dagger}\right)$ has the form:

$$
\begin{gathered}
V\left(U, U^{\dagger}, X, X^{\dagger}\right)=\frac{1}{4} \lambda_{\pi}^{2} \operatorname{Tr}\left[\left(U^{\dagger} U-\rho_{\pi} \cdot \mathbf{I}\right)^{2}\right]+\frac{1}{4} \lambda_{X}^{2}\left(X^{\dagger} X-\rho_{X}\right)^{2} \\
-\frac{B_{m}}{2 \sqrt{2}} \operatorname{Tr}\left(M U+M^{\dagger} U^{\dagger}\right)-\frac{c_{1}}{2 \sqrt{2}}\left[\operatorname{det}(U) X^{\dagger}+\operatorname{det}\left(U^{\dagger}\right) X\right] .
\end{gathered}
$$

I is the identity matrix. $M$ represents the quark mass matrix, $M=\operatorname{diag}\left(m_{1}, \ldots, m_{L}\right)$, which enters in the QCD Lagrangian as $\delta \mathcal{L}_{Q C D}^{(\text {mass })}=-\bar{q}_{R} M q_{L}-\bar{q}_{L} M^{\dagger} q_{R}$, and $A$ is the topological susceptibility in the pure-YM theory. All the parameters appearing in the Lagrangian must be considered as functions of the physical temperature $T$. In particular, the parameters $\rho_{\pi}$ and $\rho_{X}$ are responsible for the behaviour of the theory respectively across the $S U(L) \otimes S U(L)$ and the $U(1)$ chiral phase transitions, as follows:

$$
\begin{aligned}
\rho_{\pi}\left(T<T_{c h}\right) & \equiv \frac{1}{2} F_{\pi}^{2}>0, \quad \rho_{\pi}\left(T>T_{c h}\right)<0 ; \\
\rho_{X}\left(T<T_{U(1)}\right) & \equiv \frac{1}{2} F_{X}^{2}>0, \quad \rho_{X}\left(T>T_{U(1)}\right)<0 .
\end{aligned}
$$

The parameter $F_{\pi}$ is the well-known pion decay constant, while the parameter $F_{X}$ is related to the new $U(1)$ axial condensate and will be discussed at length in the rest of the paper. For $T<T_{c h}, \rho_{\pi}>0$ and therefore, by virtue of the form (4.2) of the potential, ${ }^{*}$ one finds that $\langle U\rangle \neq 0$, or, in other words $\langle\bar{q} q\rangle \neq 0$ (being $U_{i j} \sim \bar{q}_{j R} q_{i L}$, up to a multiplicative constant): i.e., the $S U(L) \otimes S U(L)$ chiral symmetry is broken. Instead, for $T>T_{c h}, \rho_{\pi}<0$ and then, always from Eq. (4.2), one has that $\langle U\rangle=0$, i.e., $\langle\bar{q} q\rangle=0$. The $U(1)$ chiral condensate stays different from zero also in the region of temperatures $T_{c h}<T<T_{U(1)}$, where, on the contrary, the $S U(L) \otimes S U(L)$ chiral symmetry is restored. In fact, for $T<T_{U(1)}, \rho_{X}>0$ and therefore, from Eq. (4.2), one finds that $\langle X\rangle \neq 0$, or, in other words, $C_{U(1)}=\left\langle\mathcal{L}_{e f f}\right\rangle \neq 0$ (being $X \sim \operatorname{det}\left(\bar{q}_{s R} q_{t L}\right)$, up to a multiplicative constant). The $U(1)$ chiral symmetry is (effectively) restored above $T_{U(1)}$, where $\rho_{X}<0$ and then, from Eq. (4.2), $\langle X\rangle=0$, i.e., $C_{U(1)}=\left\langle\mathcal{L}_{\text {eff }}\right\rangle=0$. According to what we have said in the

${ }^{*}$ As it has been stressed in Ref. [17] (see also Ref. 25]), the linear $\sigma$-type model (4.1)-44.2) contains redundant scalar fields (we are only interested in the pseudoscalar Goldstone, or would-be Goldstone, bosons), which can be eliminated by taking the limits $\lambda_{\pi}^{2}, \lambda_{X}^{2} \rightarrow+\infty$ in Eq. (4.2): so an expansion is performed not only in powers of the light quark masses $m_{i}$, but also in powers of $1 / \lambda_{\pi}^{2}$ and $1 / \lambda_{X}^{2}$. 
Introduction and in Section 2, we also assume that the topological susceptibility $A(T)$ of the pure-YM theory drops to zero at a temperature $T_{\chi}$ greater than $T_{c h}$ (but smaller than, or equal to, $\left.T_{U(1)}\right)$.

One can study the mass spectrum of the theory for $T<T_{c h}$ and $T_{c h}<T<T_{U(1)}$. First of all, let us see what happens for $T<T_{c h}$, where both the $q \bar{q}$ chiral condensate and the $U(1)$ chiral condensate are present. Integrating out the field variable $Q$ and taking only the quadratic part of the Lagrangian, one finds that, in the chiral limit $\sup \left(m_{i}\right) \rightarrow 0$, there are $L^{2}-1$ zero-mass states, which represent the $L^{2}-1$ Goldstone bosons coming from the breaking of the $S U(L) \otimes S U(L)$ chiral symmetry down to $S U(L)_{V}$. Then there are two singlet eigenstates with non-zero masses:

$$
\begin{aligned}
\eta^{\prime} & =\frac{1}{\sqrt{F_{\pi}^{2}+L F_{X}^{2}}}\left(\sqrt{L} F_{X} S_{X}+F_{\pi} S_{\pi}\right), \\
\eta_{X} & =\frac{1}{\sqrt{F_{\pi}^{2}+L F_{X}^{2}}}\left(-F_{\pi} S_{X}+\sqrt{L} F_{X} S_{\pi}\right),
\end{aligned}
$$

where $S_{\pi}$ is the usual $S U(L)$-singlet meson field associated with $U$, while $S_{X}$ is the meson field associated with $X$ (see Refs. [17, 18, 19] and Eqs. (5.4) below). The field $\eta^{\prime}$ has a "light" mass, in the sense of the $N_{c} \rightarrow \infty$ limit, being

$$
m_{\eta^{\prime}}^{2}=\frac{2 L A}{F_{\pi}^{2}+L F_{X}^{2}}=\mathcal{O}\left(\frac{1}{N_{c}}\right)
$$

This mass is intimately related to the anomaly and they both vanish in the $N_{c} \rightarrow \infty$ limit. On the contrary, the field $\eta_{X}$ has a sort of "heavy hadronic" mass of order $\mathcal{O}\left(N_{c}^{0}\right)$ in the large $-N_{c}$ limit. We immediately see that, if we put $F_{X}=0$ in the above-written formulae (i.e., if we neglect the new $U(1)$ chiral condensate), then $\eta^{\prime}=S_{\pi}$ and $m_{\eta^{\prime}}^{2}$ reduces to $\frac{2 L A}{F_{\pi}^{2}}$, which is the "usual" $\eta^{\prime}$ mass in the chiral limit [5, 6. Yet, in the general case $F_{X} \neq 0$, the two states which diagonalize the squared mass matrix are linear combinations of the "quark-antiquark" singlet field $S_{\pi}$ and of the "exotic" field $S_{X}$. Both the $\eta^{\prime}$ and the $\eta_{X}$ have the same quantum numbers (spin, parity and so on), but they have a different quark content: one is mostly $\sim i\left(\bar{q}_{L} q_{R}-\bar{q}_{R} q_{L}\right)$, while the other is $\operatorname{mostly} \sim i\left[\operatorname{det}\left(\bar{q}_{s L} q_{t R}\right)-\right.$ $\left.\operatorname{det}\left(\bar{q}_{s R} q_{t L}\right)\right]$. What happens when approaching the chiral transition temperature $T_{c h}$ ? We know that $F_{\pi}(T) \rightarrow 0$ when $T \rightarrow T_{c h}$. From Eq. (4.5) we see that $m_{\eta^{\prime}}^{2}\left(T_{c h}\right)=\frac{2 A}{F_{X}^{2}}$ and, from the first Eq. (4.4), $\eta^{\prime}\left(T_{c h}\right)=S_{X}$. We have continuity in the mass spectrum of the theory through the chiral phase transition at $T=T_{c h}$. In fact, if we study the 
mass spectrum of the theory in the region of temperatures $T_{c h}<T<T_{U(1)}$ (where the $S U(L) \otimes S U(L)$ chiral symmetry is restored, while the $U(1)$ chiral condensate is still present), one finds that there is a singlet meson field $S_{X}$ (associated with the field $X$ in the chiral Lagrangian) with a squared mass given by (in the chiral limit): $m_{S_{X}}^{2}=\frac{2 A}{F_{X}^{2}}$. This is nothing but the would-be Goldstone particle coming from the breaking of the $U(1)$ chiral symmetry, i.e., the $\eta^{\prime}$, which, for $T>T_{c h}$, is a sort of "exotic" matter field of the form $\sim i\left[\operatorname{det}\left(\bar{q}_{s L} q_{t R}\right)-\operatorname{det}\left(\bar{q}_{s R} q_{t L}\right)\right]$. Its existence could be proved perhaps in the near future by heavy-ion experiments.

\section{A relation between $\chi^{\prime}$ and the new $U(1)$ chiral condensate}

In this section and in the following one we want to describe some methods which provide us with some information about the parameter $F_{X}$. This quantity is a $U(1)$-breaking parameter: indeed, from Eq. (4.3), $\rho_{X}=\frac{1}{2} F_{X}^{2}>0$ for $T<T_{U(1)}$, and therefore, from Eq. (4.2), $\langle X\rangle=F_{X} / \sqrt{2} \neq 0$. Remembering that $X \sim \operatorname{det}\left(\bar{q}_{s R} q_{t L}\right)$, up to a multiplicative constant, we find that $F_{X}$ is proportional to the new $2 L$-fermion condensate $C_{U(1)}=\left\langle\mathcal{L}_{\text {eff }}\right\rangle$ introduced above.

In the same way, the pion decay constant $F_{\pi}$, which controls the breaking of the $S U(L) \otimes$ $S U(L)$ symmetry, is related to the $q \bar{q}$ chiral condensate by a simple and well-known proportionality relation (see Ref. [17] and references therein): $\left\langle\bar{q}_{i} q_{i}\right\rangle_{T<T_{c h}} \simeq-\frac{1}{2} B_{m} F_{\pi}$. Considering, for simplicity, the case of $L$ light quarks with the same mass $m$, one immediately derives from this equation the so-called Gell-Mann-Oakes-Renner relation [30]:

$$
m_{N S}^{2} F_{\pi}^{2} \simeq-\frac{2 m}{L}\langle\bar{q} q\rangle_{T<T_{c h}}
$$

where, as usual, $\langle\bar{q} q\rangle \equiv \sum_{i=1}^{L}\left\langle\bar{q}_{i} q_{i}\right\rangle$, and, moreover, $m_{N S}^{2}=m B_{m} / F_{\pi}, m_{N S}$ being the mass of the non-singlet pseudoscalar mesons. Eq. (5.1) relates, on the left-hand side, the pion decay constant $F_{\pi}$ and the mass $m_{N S}$ of the non-singlet mesons with, on the right-hand side, the chiral condensate $\langle\bar{q} q\rangle$ and the quark mass $m$.

It is not possible to find, in a simple way, the analogous relation between $F_{X}$ and the new condensate $C_{U(1)}=\left\langle\mathcal{L}_{e f f}\right\rangle$, since the QCD Lagrangian does not contain any term proportional to the $2 L$-fermion operator $\mathcal{L}_{\text {eff }}$. 
Alternatively, the quantity $F_{X}$ can be written in terms of a certain two-point Green function of the topological charge-density operator $Q(x)$ in the full theory with quarks. If we want to derive the two-point function of $Q(x)$, we need to consider the effective Lagrangian in the form (4.1), where the field variable $Q(x)$ has not yet been integrated. Therefore:

$$
\chi(k) \equiv-i \int d^{4} x e^{i k x}\langle T Q(x) Q(0)\rangle=\left(\mathcal{K}^{-1}(k)\right)_{11},
$$

where $\mathcal{K}^{-1}(k)$ is the inverse of the matrix $\mathcal{K}(k)$ associated with the quadratic part of the Lagrangian (4.1) in the momentum space, for the ensemble of fields $(Q(x), \ldots)$.

In particular, for $T<T_{c h}$, one has to consider the following quadratic Lagrangian, in the chiral limit $\sup \left(m_{i}\right) \rightarrow 0$ :

$$
\begin{aligned}
\mathcal{L}_{2}= & \frac{1}{2} \sum_{a=1}^{L^{2}-1} \partial_{\mu} \pi_{a} \partial^{\mu} \pi_{a}+\frac{1}{2} \partial_{\mu} S_{\pi} \partial^{\mu} S_{\pi}+\frac{1}{2} \partial_{\mu} S_{X} \partial^{\mu} S_{X} \\
& -\frac{1}{2} c\left(\frac{\sqrt{2 L}}{F_{\pi}} S_{\pi}-\frac{\sqrt{2}}{F_{X}} S_{X}\right)^{2}+\frac{1}{2 A} Q^{2} \\
& -\omega_{1} \frac{\sqrt{2 L}}{F_{\pi}} S_{\pi} Q-\left(1-\omega_{1}\right) \frac{\sqrt{2}}{F_{X}} S_{X} Q,
\end{aligned}
$$

where $c \equiv \frac{c_{1}}{\sqrt{2}}\left(\frac{F_{X}}{\sqrt{2}}\right)\left(\frac{F_{\pi}}{\sqrt{2}}\right)^{L}$. We have used for $U$ and $X$ the following exponential form, valid for $T<T_{c h}$ [17, 18, 19]:

$$
\begin{aligned}
U & =\frac{F_{\pi}}{\sqrt{2}} \exp \left(\frac{i \sqrt{2}}{F_{\pi}} \Phi\right), \quad \text { with }: \Phi=\sum_{a=1}^{L^{2}-1} \pi_{a} \tau_{a}+\frac{S_{\pi}}{\sqrt{L}} \cdot \mathbf{I} \\
X & =\frac{F_{X}}{\sqrt{2}} \exp \left(\frac{i \sqrt{2}}{F_{X}} S_{X}\right)
\end{aligned}
$$

where the matrices $\tau_{a}\left(a=1, \ldots, L^{2}-1\right)$ are the generators of the algebra of $S U(L)$ in the fundamental representation, with normalization: $\operatorname{Tr}\left(\tau_{a}\right)=0, \operatorname{Tr}\left(\tau_{a} \tau_{b}\right)=\delta_{a b}$. As already pointed out in Section $4, S_{\pi}$ is the usual "quark-antiquark" $S U(L)$-singlet meson field associated with $U$, while $S_{X}$ is the "exotic" $2 L$-fermion meson field associated with $X$. The $\pi_{a}\left(a=1, \ldots, L^{2}-1\right)$ are the self-hermitian fields describing the $L^{2}-1$ pions: they are massless in the chiral limit $\sup \left(m_{i}\right) \rightarrow 0$.

From the quadratic part of the Lagrangian (5.3) in the momentum space, we derive the following matrix $\mathcal{K}(k)$ for the ensemble of fields $\left(Q, S_{X}, S_{\pi}\right)$ [the contribution of the pion 
fields $\pi_{a}$ is simply diagonal, $\operatorname{diag}\left(k^{2}, \ldots, k^{2}\right)$, and therefore can be trivially factorized out]:

$$
\mathcal{K}(k)=\left(\begin{array}{ccc}
\frac{1}{A} & -\frac{\sqrt{2}\left(1-\omega_{1}\right)}{F_{X}} & -\frac{\omega_{1} \sqrt{2 L}}{F_{\pi}} \\
-\frac{\sqrt{2}\left(1-\omega_{1}\right)}{F_{X}} & k^{2}-\frac{2 c}{F_{X}^{2}} & \frac{2 c \sqrt{L}}{F_{\pi} F_{X}} \\
-\frac{\omega_{1} \sqrt{2 L}}{F_{\pi}} & \frac{2 c \sqrt{L}}{F_{\pi} F_{X}} & k^{2}-\frac{2 L c}{F_{\pi}^{2}}
\end{array}\right) .
$$

The calculation of the right-hand side of Eq. (5.2) can then be performed explicitly, using Eq. (5.5), obtaining:

$$
\chi(k)=\frac{A\left[k^{4}-\frac{2 c\left(F_{\pi}^{2}+L F_{X}^{2}\right)}{F_{\pi}^{2} F_{X}^{2}} k^{2}\right]}{\left[k^{4}-\frac{2 c\left(F_{\pi}^{2}+L F_{X}^{2}\right)}{F_{\pi}^{2} F_{X}^{2}} k^{2}\right]-2 A\left[\frac{\left(1-\omega_{1}\right)^{2} F_{\pi}^{2}+L \omega_{1}^{2} F_{X}^{2}}{F_{\pi}^{2} F_{X}^{2}}\right] k^{2}+\frac{4 L A c}{F_{\pi}^{2} F_{X}^{2}}} .
$$

One immediately sees that $\chi \equiv \chi(0)=0$, i.e., the topological susceptibility in the full theory with quarks vanishes in the chiral $\operatorname{limit} \sup \left(m_{i}\right) \rightarrow 0$, as expected.

But the most interesting result is found when considering the so-called "slope" of the topological susceptibility, defined as:

$$
\left.\chi^{\prime} \equiv \frac{1}{8} \frac{\partial}{\partial k_{\mu}} \frac{\partial}{\partial k^{\mu}} \chi(k)\right|_{k=0}=\frac{i}{8} \int d^{4} x x^{2}\langle T Q(x) Q(0)\rangle .
$$

Moreover, whenever $\chi(k)$ is a function of $k^{2}$ (such as in the theory at $T=0$, by virtue of the Lorentz invariance), one can also write:

$$
\chi^{\prime}=\left.\frac{d}{d k^{2}} \chi(k)\right|_{k=0}
$$

In the theory at finite temperature $T \neq 0$ the Lorentz invariance is broken down to the $O(3)$ invariance under spatial rotations only, so that $\chi(k)$ is, in general, a function of $\vec{k}^{2}$ and $k^{0}$. However, the propagator $\chi(k)$ obtained in Eq. (5.6) is a function of $k^{2}=\left(k^{0}\right)^{2}-\vec{k}^{2}$ also in the case of non-zero temperature. Therefore, we can explicitly calculate the quantity $\chi^{\prime}$ in our effective model in the chiral limit $\sup \left(m_{i}\right) \rightarrow 0$ (and we shall call " $\chi_{c h}^{\prime}$ " its value), using Eqs. (5.6) and (5.8), obtaining:

$$
\chi_{c h}^{\prime}=-\frac{1}{2 L}\left(F_{\pi}^{2}+L F_{X}^{2}\right) \equiv-\frac{1}{2 L} F_{\eta^{\prime}}^{2},
$$


where $F_{\eta^{\prime}} \equiv \sqrt{F_{\pi}^{2}+L F_{X}^{2}}$ is the decay constant of the $\eta^{\prime}$ (at the leading order in the $1 / N_{c}$ expansion), modified by the presence of the new $U(1)$ chiral order parameter [19].

For the benefit of the reader, we here briefly repeat the arguments developed in Ref. [19], leading to the result $F_{\eta^{\prime}} \equiv \sqrt{F_{\pi}^{2}+L F_{X}^{2}}$ : this will also provide us with an alternative derivation of Eq. (5.9). We shall consider the $T=0$ case for simplicity.

It turns out that $\eta^{\prime}$ is just the meson state, with a squared mass of order $1 / N_{c}$, whose contribution to the full topological susceptibility $\chi$ exactly cancels out (in the chiral limit of massless quarks) the pure-gauge part $A$ of $\chi$, so making $\chi=0$ : this is the socalled Witten's mechanism. To see how this picture comes out in our theory, one first determines the $U(1)$ axial current, starting from our effective Lagrangian. This is easily done remembering how the fields $U$ and $X$ transform under a $U(1)$ chiral transformation and one ends up with the following expression [19, 20]:

$$
J_{5, \mu}=i\left[\operatorname{Tr}\left(U^{\dagger} \partial_{\mu} U-U \partial_{\mu} U^{\dagger}\right)+L\left(X^{\dagger} \partial_{\mu} X-X \partial_{\mu} X^{\dagger}\right)\right]
$$

After having inserted here the expressions (5.4) in place of $U$ and $X$, the current $J_{5, \mu}$ takes the following form:

$$
J_{5, \mu}=-\sqrt{2 L} F_{\eta^{\prime}} \partial_{\mu} \eta^{\prime}
$$

where the field $\eta^{\prime}$ is defined by the first Eq. (4.4) and the relative coupling between $J_{5, \mu}$ and $\eta^{\prime}$, i.e., the $S U(L)$-singlet $\left(\eta^{\prime}\right)$ decay constant defined as $\left\langle 0\left|J_{5, \mu}(0)\right| \eta^{\prime}(p)\right\rangle=i \sqrt{2 L} p_{\mu} F_{\eta^{\prime}}$, is given by:

$$
F_{\eta^{\prime}}=\sqrt{F_{\pi}^{2}+L F_{X}^{2}}
$$

Let us now recall the Witten's argument and write the two-point function (at fourmomentum $k$ ) of the topological charge density $Q(x)$ as a sum over one-hadron poles, i.e., one-hadron intermediate states:

$$
\chi(k)=-i \int d^{4} x e^{i k x}\langle T Q(x) Q(0)\rangle=A_{0}(k)+\sum_{\text {mesons }} \frac{|\langle 0|Q| n\rangle|^{2}}{k^{2}-m_{n}^{2}}
$$

where $A_{0}(k)$ is the pure Yang-Mills contribution from the glueball intermediate states and it is the leading-order term in $1 / N_{c}$ [being of order $\mathcal{O}\left(N_{c}^{0}\right)$ ]. In the chiral limit in which we have $L$ massless quarks, the full topological susceptibility $\chi \equiv \chi(k=0)$ must vanish: so there must be a meson state, with squared mass $m_{n}^{2}=\mathcal{O}\left(1 / N_{c}\right)$ [since $A_{0}(0)=\mathcal{O}\left(N_{c}^{0}\right)$, 
while $|\langle 0|Q| n\rangle|^{2}=\mathcal{O}\left(1 / N_{c}\right)$ ], which exactly cancels out $A_{0}(0)$. This is the meson that we usually call $\eta^{\prime}$ : the other meson states in (5.13) have squared masses of order $\mathcal{O}\left(N_{c}^{0}\right)$, so that their contributions to the summation in (5.13) are suppressed by a factor of $1 / N_{c}$. Therefore we obtain that:

$$
\frac{\left|\left\langle 0|Q| \eta^{\prime}\right\rangle\right|^{2}}{m_{\eta^{\prime}}^{2}}=A
$$

where $A \equiv A_{0}(0)$ is the pure Yang-Mills topological susceptibility in the large $-N_{c}$ limit. In the chiral limit of $L$ massless quarks, the topological charge density $Q(x)$ is directly related to the four-divergence of the axial current $J_{5, \mu}$, via the anomaly equation, $\partial^{\mu} J_{5, \mu}(x)=$ $2 L Q(x)$, so that $\left\langle 0|Q| \eta^{\prime}\right\rangle=\frac{1}{\sqrt{2 L}} m_{\eta^{\prime}}^{2} F_{\eta^{\prime}}$, which can be substituted into Eq. (5.14) to give:

$$
A=\frac{m_{\eta^{\prime}}^{2} F_{\eta^{\prime}}^{2}}{2 L}
$$

This equation relates the mass $m_{\eta^{\prime}}$ of the $\eta^{\prime}$ state, its decay constant $F_{\eta^{\prime}}$ and the puregauge topological susceptibility $A$. And in fact Eq. (15.15) is verified when putting for $m_{\eta^{\prime}}$ and $F_{\eta^{\prime}}$ their values determined above in Eqs. (4.5) and (5.12).

The dominance of the $\eta^{\prime}$ state in the sum over the meson states at the right-hand side of Eq. (5.13) can also be used to evaluate the slope of the topological susceptibility:

$$
\chi_{c h}^{\prime}=\left.\frac{d}{d k^{2}} \chi(k)\right|_{k=0} \simeq-\frac{\left|\left\langle 0|Q(0)| \eta^{\prime}\right\rangle\right|^{2}}{m_{\eta^{\prime}}^{4}}=-\frac{1}{2 L} F_{\eta^{\prime}}^{2}=-\frac{1}{2 L}\left(F_{\pi}^{2}+L F_{X}^{2}\right),
$$

thus recovering the result (5.9), derived above from our effective Lagrangian. This is perfectly natural: using the effective Lagrangian at tree-level (i.e., using its "free" propagators) one gets the results in the one-hadron pole (i.e., one-hadron intermediate state) approximation.

Summarizing, we have found that the value of $\chi^{\prime}$ in the chiral limit $\sup \left(m_{i}\right) \rightarrow 0$ is shifted from the "original" value $-\frac{1}{2 L} F_{\pi}^{2}$ (derived in the absence of an extra $U(1)$ chiral condensate: see Refs. [31, where $\chi_{c h}^{\prime}$ is shown to be a relevant quantity in the discussion of the so-called "proton-spin crisis" problem, and also Ref. [32]) to the value $-\frac{1}{2 L} F_{\eta^{\prime}}^{2}=-\frac{1}{2 L}\left(F_{\pi}^{2}+L F_{X}^{2}\right)$, which also depends on the quantity $F_{X}$, proportional to the extra $U(1)$ chiral condensate.

Therefore, a measure of this quantity $\chi_{c h}^{\prime}$, e.g., in lattice gauge theory, could provide an estimate for the $\eta^{\prime}$ decay constant $F_{\eta^{\prime}}$, and, as a consequence, for $F_{X}$. At present, lattice 
determinations of $\chi^{\prime}$ only exist for the pure-gauge theory at $T=0$, with gauge group $S U(2)[33$ and $S U(3)[34 . *$

All the above refers to the theory at $T=0$ (or, more generally, for $T<T_{c h}$ ).

When approaching the chiral transition at $T=T_{c h}$, one expects that $F_{\pi}$ vanishes, while $F_{X}$ remains different from zero and the quantity $\chi_{c h}^{\prime}$ tends to the value:

$$
\chi_{c h}^{\prime} \underset{T \rightarrow T_{c h}}{\longrightarrow}-\frac{1}{2} F_{X}^{2}
$$

Indeed, the quantity $\chi(k)=-i \int d^{4} x e^{i k x}\langle T Q(x) Q(0)\rangle$ can also be evaluated in the region of temperatures $T_{c h}<T<T_{U(1)}$, proceeding as for the case $T<T_{c h}$, obtaining the result (already derived in Ref. [17]):

$$
\chi(k)=A \frac{k^{2}}{k^{2}-\frac{2 A}{F_{X}^{2}}},
$$

in the chiral limit $\sup \left(m_{i}\right) \rightarrow 0$.

Therefore, in the region of temperatures $T_{c h}<T<T_{U(1)}, \chi_{c h}^{\prime}$ is given by:

$$
\chi_{c h}^{\prime}=\left.\frac{d}{d k^{2}} \chi(k)\right|_{k=0}=-\frac{1}{2} F_{X}^{2},
$$

consistently with the results (5.9) and (5.17) found above: i.e., $\chi_{c h}^{\prime}$ varies with continuity across $T_{c h}$. This means that $\chi_{c h}^{\prime}$ acts as a sort of order parameter for the $U(1)$ axial symmetry above $T_{c h}$ : if $\chi_{c h}^{\prime}$ is different from zero above $T_{c h}$, this means that the $U(1)-$ breaking parameter $F_{X}$ is different from zero.

\section{Radiative decays of the pseudoscalar mesons}

Further information on the quantity $F_{X}$ (i.e., on the new $U(1)$ chiral condensate, to which it is related) can be derived from the study of the radiative decays of the "light" pseudoscalar mesons in two photons. These decays were also studied in Ref. [35], using an

${ }^{*}$ It has been recently pointed out in Ref. 32] that there can be ambiguities in the definition of $\chi_{c h}^{\prime}$ in a lattice regularized theory. However, these ambiguities do not apply to the leading term [of order $\mathcal{O}\left(N_{c}\right)$ ] given by Eq. (5.9), but they can affect the next-to-leading term [of order $\mathcal{O}\left(N_{c}^{0}\right)$ ] in the $1 / N_{c}$ expansion. 
effective Lagrangian model, in which only the $q \bar{q}$ chiral condensate was considered. In this section we want to find the decay rates of the processes $\pi^{0}, \eta, \eta^{\prime}, \eta_{X} \rightarrow \gamma \gamma$ (where $\eta_{X}$ represents the pseudoscalar meson state, introduced in Section 4, having the same quantum numbers of the $\eta^{\prime}$, but a larger mass and a different quark content) and see which are the effects due to the new $U(1)$ axial condensate, in the realistic case of $L=3$ light quarks and in the simple case of zero temperature $(T=0)$.

To this purpose, we have to introduce the electromagnetic interaction in our effective model. First of all, in order to make the Lagrangian invariant under local $U(1)$ electromagnetic transformations $\left[q \rightarrow q^{\prime}=e^{i \theta e \mathbf{Q}} q\right.$, in terms of the quark fields; the matrix $\mathbf{Q}$ is defined in Eq. (6.2) below], we have to replace the derivative of the field $U$ with the corresponding covariant derivative $D_{\mu} U$, which, by virtue of the transformation property of the field $U\left[U \rightarrow U^{\prime}=e^{i \theta e \mathbf{Q}} U e^{-i \theta e \mathbf{Q}}\right]$, has the following form:

$$
D_{\mu} U=\partial_{\mu} U+i e A_{\mu}[\mathbf{Q}, U]
$$

where $A_{\mu}$ is the electromagnetic field (which transforms as: $A_{\mu} \rightarrow A_{\mu}^{\prime}=A_{\mu}-\partial_{\mu} \theta$ ) and $\mathrm{Q}$ is the quark charge matrix (in units of $e$, the absolute value of the electron charge):

$$
\mathbf{Q}=\left(\begin{array}{lll}
\frac{2}{3} & & \\
& -\frac{1}{3} & \\
& & -\frac{1}{3}
\end{array}\right)
$$

Instead,the field $X$ is invariant under a $U(1)$ electromagnetic gauge transformation and therefore its covariant derivative just coincides with the ordinary four-derivative: $D_{\mu} X=$ $\partial_{\mu} X$.

In addition, we have to reproduce the effects of the electromagnetic anomaly, whose contribution to the four-divergence of the $U(1)$ axial current $\left(J_{5, \mu}=\bar{q} \gamma_{\mu} \gamma_{5} q\right)$ and of the $S U(3)$ axial currents $\left(A_{\mu}^{a}=\bar{q} \gamma_{\mu} \gamma_{5} \frac{\tau_{a}}{\sqrt{2}} q\right.$, where the matrices $\tau_{a}$, with $a=1, \ldots, 8$, are the generators of the algebra of $S U(3)$ in the fundamental representation, already introduced in the previous section) is given by:

$$
\left(\partial^{\mu} J_{5, \mu}\right)_{\text {anomaly }}^{\text {e.m. }}=2 \operatorname{Tr}\left(\mathbf{Q}^{2}\right) G, \quad\left(\partial^{\mu} A_{\mu}^{a}\right)_{\text {anomaly }}^{\text {e.m. }}=2 \operatorname{Tr}\left(\mathbf{Q}^{2} \frac{\tau_{a}}{\sqrt{2}}\right) G
$$

where $G \equiv \frac{e^{2} N_{c}}{32 \pi^{2}} \varepsilon^{\mu \nu \rho \sigma} F_{\mu \nu} F_{\rho \sigma}$ ( $F_{\mu \nu}$ being the electromagnetic field-strength tensor), thus breaking the corresponding chiral symmetries. We observe that $\operatorname{Tr}\left(\mathbf{Q}^{2} \tau_{a}\right) \neq 0$ only for 
$a=3$ or $a=8$.

We must look for an interaction term $\mathcal{L}_{I}$ (constructed with the chiral Lagrangian fields and the electromagnetic operator $G$ ) which, under a $U(1)$ axial transformation $q \rightarrow q^{\prime}=$ $e^{-i \alpha \gamma_{5}} q$, transforms as:

$$
U(1)_{A}: \quad \mathcal{L}_{I} \rightarrow \mathcal{L}_{I}+2 \alpha \operatorname{Tr}\left(\mathbf{Q}^{2}\right) G
$$

while, under $S U(3)$ axial transformations of the type $q \rightarrow q^{\prime}=e^{-i \beta \gamma_{5} \tau_{a} / \sqrt{2}} q$ (with $a=3,8$ ), transforms as:

$$
S U(3)_{A}: \quad \mathcal{L}_{I} \rightarrow \mathcal{L}_{I}+2 \beta \operatorname{Tr}\left(\mathrm{Q}^{2} \frac{\tau_{a}}{\sqrt{2}}\right) G .
$$

The electromagnetic anomaly term for the field $U$, originally proposed in Ref. [35, has the following form: $\frac{1}{2} i G \operatorname{Tr}\left[\mathbf{Q}^{2}\left(\ln U-\ln U^{\dagger}\right)\right]$. By virtue of the transformation property of the field $U$ under a $U(L) \otimes U(L)$ chiral transformation $\left(q_{L} \rightarrow V_{L} q_{L}, q_{R} \rightarrow V_{R} q_{R} \Rightarrow\right.$ $U \rightarrow V_{L} U V_{R}^{\dagger}$, where $V_{L}$ and $V_{R}$ are arbitrary unitary matrices [17, 20]), it is immediate to see that this term satisfies both the transformation properties (6.4) and (6.5).

If we also consider an analogous term for the field $X$, of the form $\frac{1}{2 L} i G \operatorname{Tr}\left(\mathbf{Q}^{2}\right)\left(\ln X-\ln X^{\dagger}\right)$ (where, in our case, $L=3$ ), then, by virtue of the transformation property of the field $X$ under a $U(L) \otimes U(L)$ chiral transformation $\left(X \rightarrow \operatorname{det}\left(V_{L}\right) \operatorname{det}\left(V_{R}\right)^{*} X\right.$ [17, 20] $)$, one can see that this term satisfies the transformation property (6.4) under a $U(1)$ axial transformation, while it is invariant under $S U(3)$ axial transformations. Therefore, if we try to consider a linear combination of the two terms (inspired by what was done for the $U(1)$ axial anomaly terms in Eq. (4.11) [17]), i.e.,

$$
\mathcal{L}_{I}=\frac{1}{2} i \omega_{2} G \operatorname{Tr}\left[\mathbf{Q}^{2}\left(\ln U-\ln U^{\dagger}\right)\right]+\frac{1}{2 L} i\left(1-\omega_{2}\right) G \operatorname{Tr}\left(\mathbf{Q}^{2}\right)\left(\ln X-\ln X^{\dagger}\right),
$$

we immediately see that the property (6.4) is satisfied for every value of the parameter $\omega_{2}$, while the property (6.5) is satisfied only for $\omega_{2}=1$.

In conclusion, the electromagnetic anomaly interaction term is simply given by:

$$
\mathcal{L}_{I}=\frac{1}{2} i G \operatorname{Tr}\left[\mathbf{Q}^{2}\left(\ln U-\ln U^{\dagger}\right)\right]
$$

Therefore, we have to consider the following effective chiral Lagrangian, which includes the electromagnetic interaction terms described above:

$$
\mathcal{L}\left(U, U^{\dagger}, X, X^{\dagger}, Q, A^{\mu}\right)=\frac{1}{2} \operatorname{Tr}\left(D_{\mu} U D^{\mu} U^{\dagger}\right)+\frac{1}{2} \partial_{\mu} X \partial^{\mu} X^{\dagger}
$$




$$
\begin{aligned}
& -V\left(U, U^{\dagger}, X, X^{\dagger}\right)+\frac{1}{2} i Q \omega_{1} \operatorname{Tr}\left(\ln U-\ln U^{\dagger}\right) \\
& +\frac{1}{2} i Q\left(1-\omega_{1}\right)\left(\ln X-\ln X^{\dagger}\right)+\frac{1}{2 A} Q^{2} \\
& +\frac{1}{2} i G \operatorname{Tr}\left[\mathbf{Q}^{2}\left(\ln U-\ln U^{\dagger}\right)\right]-\frac{1}{4} F_{\mu \nu} F^{\mu \nu}
\end{aligned}
$$

where the potential term $V\left(U, U^{\dagger}, X, X^{\dagger}\right)$ is the one written in Eq. (4.2).

We can now describe the predictions of our model about the decay amplitudes and rates of the processes $\pi^{0}, \eta, \eta^{\prime}, \eta_{X} \rightarrow \gamma \gamma$. It is well known that the two final photons in the pseudoscalar-meson decays are associated with the pseudoscalar operator $\varepsilon^{\mu \nu \rho \sigma} F_{\mu \nu} F_{\rho \sigma}$ : so, e.g., the $\pi^{0}$ decay is reproduced by an interaction of the form $\pi_{3} \varepsilon^{\mu \nu \rho \sigma} F_{\mu \nu} F_{\rho \sigma}$. The covariant derivative does not contain this term (since the diagonal matrix $\mathbf{Q}$ and the generator $\tau_{3}$ commute); instead, it is produced by the electromagnetic anomaly interaction written in Eq. (6.7) (indeed the chiral symmetry is broken by the anomaly). The same arguments can be applied to the other decays. Therefore, the decay amplitude of the generic process "meson $\rightarrow \gamma \gamma$ " is given by:

$$
A(\text { meson } \rightarrow \gamma \gamma)=\left\langle\gamma \gamma\left|\mathcal{L}_{I}(0)\right| \text { meson }\right\rangle
$$

Substituting the exponential form (5.4) (with $L=3$ ) of the field $U$ (we remind the reader that we are considering the case of zero temperature) into Eq. (6.7), we have the following interaction Lagrangian:

$$
\mathcal{L}_{I}=-G \frac{1}{3 F_{\pi}}\left(\pi_{3}+\frac{1}{\sqrt{3}} \pi_{8}+\frac{2 \sqrt{2}}{\sqrt{3}} S_{\pi}\right) .
$$

We immediately consider the realistic case of $L=3$ quarks with masses different from zero. In this case one has to consider the following quadratic Lagrangian, which can be obtained substituting the exponential expressions (15.4) of the fields $U$ and $X$ into Eq. (4.1):

$$
\begin{aligned}
\mathcal{L}_{2} & =\frac{1}{2} \operatorname{Tr}\left(\partial_{\mu} \Phi \partial^{\mu} \Phi\right)+\frac{1}{2} \partial_{\mu} S_{X} \partial^{\mu} S_{X}-\frac{B_{m}}{2 F_{\pi}} \operatorname{Tr}\left(M \Phi^{2}\right) \\
& -c\left(\frac{1}{F_{\pi}} \operatorname{Tr} \Phi-\frac{1}{F_{X}} S_{X}\right)^{2}-A\left(\frac{\omega_{1}}{F_{\pi}} \operatorname{Tr} \Phi+\frac{1-\omega_{1}}{F_{x}} S_{X}\right)^{2}
\end{aligned}
$$

where $c \equiv \frac{c_{1}}{\sqrt{2}}\left(\frac{F_{X}}{\sqrt{2}}\right)\left(\frac{F_{\pi}}{\sqrt{2}}\right)^{3}$.

Substituting Eq. (15.4) (with $L=3$ ) into Eq. (6.11), one immediately sees that the fields 
$\pi_{1}, \pi_{2}, \pi_{4}, \pi_{5}, \pi_{6}, \pi_{7}$ are diagonal, while the fields $\pi_{3}, \pi_{8}, S_{\pi}, S_{X}$ mix together. However, neglecting the experimentally small mass difference between the quarks up and down (i.e., neglecting the experimentally small violations of the $S U(2)$ isotopic spin), also $\pi_{3}$ becomes diagonal and can be identified with the physical state $\pi^{0}$. The other physical states can be found by the diagonalization of the following squared mass matrix, written for the ensemble of fields $\left(\pi_{8}, S_{\pi}, S_{X}\right)$ (originally derived in Ref. [19]):

$$
\mathcal{K}=\left(\begin{array}{ccc}
\frac{2 B}{3}\left(\tilde{m}+2 m_{s}\right) & \frac{2 B \sqrt{2}}{3}\left(\tilde{m}-m_{s}\right) & 0 \\
\frac{2 B \sqrt{2}}{3}\left(\tilde{m}-m_{s}\right) & \frac{6\left(A \omega_{1}^{2}+c\right)}{F_{\pi}^{2}}+\tilde{m}_{0}^{2} & \frac{2 \sqrt{3}\left[A \omega_{1}\left(1-\omega_{1}\right)-c\right]}{F_{\pi} F_{X}} \\
0 & \frac{2 \sqrt{3}\left[A \omega_{1}\left(1-\omega_{1}\right)-c\right]}{F_{\pi} F_{X}} & \frac{2\left[A\left(1-\omega_{1}\right)^{2}+c\right]}{F_{X}^{2}}
\end{array}\right)
$$

where:

$$
B \equiv \frac{B_{m}}{2 F_{\pi}}, \quad \tilde{m} \equiv \frac{m_{u}+m_{d}}{2}, \quad \tilde{m}_{0}^{2} \equiv \frac{2}{3} B\left(2 \tilde{m}+m_{s}\right) .
$$

The fields $\left(\pi_{8}, S_{\pi}, S_{X}\right)$ can be written in terms of the eigenstates $\eta, \eta^{\prime}, \eta_{X}$ as follows:

$$
\left(\begin{array}{c}
\pi_{8} \\
S_{\pi} \\
S_{X}
\end{array}\right)=\mathbf{C}\left(\begin{array}{c}
\eta \\
\eta^{\prime} \\
\eta_{X}
\end{array}\right)
$$

where $\mathbf{C}$ is the following $3 \times 3$ orthogonal matrix:

$$
\mathbf{C}=\left(\begin{array}{ccc}
\alpha_{1} & \alpha_{2} & \alpha_{3} \\
\beta_{1} & \beta_{2} & \beta_{3} \\
\gamma_{1} & \gamma_{2} & \gamma_{3}
\end{array}\right)=\left(\begin{array}{ccc}
\cos \tilde{\varphi} & -\sin \tilde{\varphi} & 0 \\
\sin \tilde{\varphi} \frac{F_{\pi}}{\sqrt{F_{\pi}^{2}+3 F_{X}^{2}}} & \cos \tilde{\varphi} \frac{F_{\pi}}{\sqrt{F_{\pi}^{2}+3 F_{X}^{2}}} & \frac{\sqrt{3} F_{X}}{\sqrt{F_{\pi}^{2}+3 F_{X}^{2}}} \\
\sin \tilde{\varphi} \frac{\sqrt{3} F_{X}}{\sqrt{F_{\pi}^{2}+3 F_{X}^{2}}} & \cos \tilde{\varphi} \frac{\sqrt{3} F_{X}}{\sqrt{F_{\pi}^{2}+3 F_{X}^{2}}} & -\frac{F_{\pi}}{\sqrt{F_{\pi}^{2}+3 F_{X}^{2}}}
\end{array}\right)
$$

Here $\tilde{\varphi}$ is a mixing angle, which can be related to the masses of the quarks up, down, strange (and therefore to the masses of the octet mesons) by the following relation:

$$
\tan \tilde{\varphi}=\frac{\sqrt{2}}{9 A} B F_{\pi} \sqrt{F_{\pi}^{2}+3 F_{X}^{2}}\left(m_{s}-\tilde{m}\right)=\frac{F_{\pi} \sqrt{F_{\pi}^{2}+3 F_{X}^{2}}}{6 \sqrt{2} A}\left(m_{\eta}^{2}-m_{\pi}^{2}\right),
$$


where $m_{\pi}^{2}=2 B \tilde{m}$ and $m_{\eta}^{2}=\frac{2}{3} B\left(\tilde{m}+2 m_{s}\right)$. The masses $m_{\eta}, m_{\eta^{\prime}}, m_{\eta_{X}}$ are given by the squared root of the eigenvalues of the squared mass matrix written in Eq. (6.12) [19].

The interaction Lagrangian (6.10), written in terms of the physical fields $\pi^{0}, \eta, \eta^{\prime}$ and $\eta_{X}$, reads as follows:

$$
\mathcal{L}_{I} \equiv-G \frac{1}{3 F_{\pi}}\left(\pi^{0}+a_{1} \eta+a_{2} \eta^{\prime}+a_{3} \eta_{X}\right)
$$

where $a_{i}=\frac{1}{\sqrt{3}}\left(\alpha_{i}+2 \sqrt{2} \beta_{i}\right)$ (for $\left.i=1,2,3\right)$, so that:

$$
\begin{aligned}
& a_{1}=\sqrt{\frac{1}{3}}\left(\cos \tilde{\varphi}+2 \sqrt{2} \sin \tilde{\varphi} \frac{F_{\pi}}{\sqrt{F_{\pi}^{2}+3 F_{X}^{2}}}\right), \\
& a_{2}=\sqrt{\frac{1}{3}}\left(2 \sqrt{2} \cos \tilde{\varphi} \frac{F_{\pi}}{\sqrt{F_{\pi}^{2}+3 F_{X}^{2}}}-\sin \tilde{\varphi}\right), \\
& a_{3}=2 \sqrt{2}\left(\frac{F_{X}}{\sqrt{F_{\pi}^{2}+3 F_{X}^{2}}}\right) .
\end{aligned}
$$

With simple calculations we find the following expressions for the decay amplitudes:

$$
\begin{aligned}
A\left(\pi^{0} \rightarrow \gamma \gamma\right) & =\frac{e^{2} N_{c}}{12 \pi^{2} F_{\pi}} I, \\
A(\eta \rightarrow \gamma \gamma) & =\frac{e^{2} N_{c}}{12 \pi^{2} F_{\pi}} \sqrt{\frac{1}{3}}\left(\cos \tilde{\varphi}+2 \sqrt{2} \sin \tilde{\varphi} \frac{F_{\pi}}{\sqrt{F_{\pi}^{2}+3 F_{X}^{2}}}\right) I, \\
A\left(\eta^{\prime} \rightarrow \gamma \gamma\right) & =\frac{e^{2} N_{c}}{12 \pi^{2} F_{\pi}} \sqrt{\frac{1}{3}}\left(2 \sqrt{2} \cos \tilde{\varphi} \frac{F_{\pi}}{\sqrt{F_{\pi}^{2}+3 F_{X}^{2}}}-\sin \tilde{\varphi}\right) I, \\
A\left(\eta_{X} \rightarrow \gamma \gamma\right) & =\frac{e^{2} N_{c}}{12 \pi^{2} F_{\pi}} 2 \sqrt{2}\left(\frac{F_{X}}{\sqrt{F_{\pi}^{2}+3 F_{X}^{2}}}\right) I,
\end{aligned}
$$

where $I \equiv \varepsilon_{\mu \nu \rho \sigma} k_{1}^{\mu} \epsilon_{1}^{\nu *} k_{2}^{\rho} \epsilon_{2}^{\sigma *}\left(k_{1}, k_{2}\right.$ being the four-momenta of the two final photons and $\epsilon_{1}, \epsilon_{2}$ their polarizations). Consequently we derive the following decay rates (in the real case $N_{c}=3$ ):

$$
\begin{aligned}
\Gamma\left(\pi^{0} \rightarrow \gamma \gamma\right) & =\frac{\alpha^{2} m_{\pi}^{3}}{64 \pi^{3} F_{\pi}^{2}}, \\
\Gamma(\eta \rightarrow \gamma \gamma) & =\frac{\alpha^{2} m_{\eta}^{3}}{192 \pi^{3} F_{\pi}^{2}}\left(\cos \tilde{\varphi}+2 \sqrt{2} \sin \tilde{\varphi} \frac{F_{\pi}}{\sqrt{F_{\pi}^{2}+3 F_{X}^{2}}}\right)^{2},
\end{aligned}
$$




$$
\begin{aligned}
\Gamma\left(\eta^{\prime} \rightarrow \gamma \gamma\right) & =\frac{\alpha^{2} m_{\eta^{\prime}}^{3}}{192 \pi^{3} F_{\pi}^{2}}\left(2 \sqrt{2} \cos \tilde{\varphi} \frac{F_{\pi}}{\sqrt{F_{\pi}^{2}+3 F_{X}^{2}}}-\sin \tilde{\varphi}\right)^{2}, \\
\Gamma\left(\eta_{X} \rightarrow \gamma \gamma\right) & =\frac{\alpha^{2} m_{\eta_{X}}^{3}}{8 \pi^{3} F_{\pi}^{2}}\left(\frac{F_{X}}{\sqrt{F_{\pi}^{2}+3 F_{X}^{2}}}\right)^{2},
\end{aligned}
$$

where $\alpha=e^{2} / 4 \pi \simeq 1 / 137$ is the fine-structure constant.

If we put $F_{X}=0$ (i.e., if we neglect the new $U(1)$ chiral condensate), the expressions $(6.22) \div 6.23$ ) written above reduce to the corresponding amplitudes derived in Ref. 35] using an effective Lagrangian which includes only the usual $q \bar{q}$ chiral condensate (so there is no field $\eta_{X}$ !):

$$
\begin{aligned}
A(\eta \rightarrow \gamma \gamma) & =\frac{e^{2} N_{c}}{12 \pi^{2} F_{\pi}} \sqrt{\frac{1}{3}}(\cos \varphi+2 \sqrt{2} \sin \varphi) I \\
A\left(\eta^{\prime} \rightarrow \gamma \gamma\right) & =\frac{e^{2} N_{c}}{12 \pi^{2} F_{\pi}} \sqrt{\frac{1}{3}}(2 \sqrt{2} \cos \varphi-\sin \varphi) I
\end{aligned}
$$

The mixing angle $\varphi$ is now defined as follows [see Eqs. (6.14)-(6.15) with $F_{X}=0$ ]:

$$
\begin{aligned}
\pi_{8} & =\eta \cos \varphi-\eta^{\prime} \sin \varphi \\
S_{\pi} & =\eta \sin \varphi+\eta^{\prime} \cos \varphi
\end{aligned}
$$

and is related to the quark masses (i.e., to the masses of the octet mesons) by the following relation [see Eq. (6.16) with $F_{X}=0$ ]:

$$
\tan \varphi \simeq \frac{\sqrt{2}}{9 A} B F_{\pi}^{2}\left(m_{s}-\tilde{m}\right)=\frac{F_{\pi}^{2}}{6 \sqrt{2} A}\left(m_{\eta}^{2}-m_{\pi}^{2}\right)
$$

Therefore, the introduction of the new condensate (while leaving the $\pi^{0} \rightarrow \gamma \gamma$ decay rate unaffected, as it must!) modifies the decay rates of $\eta$ and $\eta^{\prime}$ (and, moreover, we also have to consider the particle $\eta_{X}$ ). In particular, it modifies the $\eta^{\prime}$ decay constant, already in the chiral limit $\sup \left(m_{i}\right) \rightarrow 0$. Indeed, in this limit, Eq. (6.30) [with $\varphi=0$ : see Eq. (6.32)] differs from the corresponding Eq. (6.23) [with $\tilde{\varphi}=0$ : see Eq. (6.16)] by the substitution of the pion decay constant $F_{\pi}$ with the quantity $F_{\eta^{\prime}} \equiv \sqrt{F_{\pi}^{2}+3 F_{X}^{2}}$, which (as it has been explained in the previous section for the general case of $L$ light flavours) can be identified with the $\eta^{\prime}$ decay constant (in the large $-N_{c}$ limit).

In conclusion, a study of the radiative decays $\eta \rightarrow \gamma \gamma, \eta^{\prime} \rightarrow \gamma \gamma$ and a comparison with the experimental data can provide us with further information about the parameter $F_{X}$ 
and the new exotic condensate. For example, from Eqs. (6.26) and (6.27), and using the experimental values for the various quantities which there appear, i.e.,

$$
\begin{aligned}
& F_{\pi}=92.4(4) \mathrm{MeV}, \\
& m_{\eta}=547.30(12) \mathrm{MeV}, \\
& m_{\eta^{\prime}}=957.78(14) \mathrm{MeV}, \\
& \Gamma(\eta \rightarrow \gamma \gamma)=0.46(4) \mathrm{KeV}, \\
& \Gamma\left(\eta^{\prime} \rightarrow \gamma \gamma\right)=4.26(19) \mathrm{KeV},
\end{aligned}
$$

we can extract the following values for the quantity $F_{X}$ and for the mixing angle $\tilde{\varphi}$ :

$$
F_{X}=27(9) \mathrm{MeV}, \quad \tilde{\varphi}=16(3)^{0} .
$$

The value of $F_{X}$ is not far from the upper limit $\left|F_{X}\right| \lesssim 20 \mathrm{MeV}$ obtained from the generalized Witten-Veneziano formula derived in Ref. [19]. Moreover, the values of $F_{X}$ and $\tilde{\varphi}$ so found are perfectly consistent with the relation (6.16) for the mixing angle.* We thus see that $F_{X}$ is small when compared with $F_{\pi}$. In particular, the corrections due to the presence of the new $U(1)$ condensate are proportional to the ratio $F_{X}^{2} / F_{\pi}^{2}$ [see Eqs. $(6.16)$, and $(6.26) \div(6.28)]$ and they are of the order of some $\%$.

\section{Conclusions}

In this paper we have discussed the role of the $U(1)$ axial symmetry in QCD both at zero and at finite temperature. One expects that, above a certain critical temperature $T_{U(1)}$, also the $U(1)$ axial symmetry will be (effectively) restored. We have tried to see if this transition has (or has not) anything to do with the usual $S U(L) \otimes S U(L)$ chiral transition: various possible scenarios have been discussed in Section 2. In particular, supported by recent lattice results on the pure-YM topological susceptibility and the socalled "chiral susceptibilities" (which have been discussed at length in the Introduction), we have analysed a scenario in which a new $U(1)$-breaking condensate survives across

${ }^{*}$ We use for the pure-YM topological susceptibility the value $A=(180 \pm 5 \mathrm{MeV})^{4}$, obtained from lattice simulations [36, 37, 11]. 
the chiral transition at $T_{c h}$, staying different from zero up to $T_{U(1)}>T_{c h}$. This scenario can be consistently reproduced using an effective Lagrangian model, which also includes the new $U(1)$ chiral condensate: this theoretical model was originally proposed in Refs. [17, 18, 19] and it has been briefly summarized in Sections 3 and 4 for the convenience of the reader. This scenario could perhaps be verified in the near future by heavy-ion experiments, by analysing the pseudoscalar-meson spectrum in the singlet sector.

In Section 5 (which, together with Section 6, contains the main original results of this paper) we have analysed the consequences of our theoretical model on the slope of the topological susceptibility $\chi^{\prime}$, in the full theory with quarks, showing how this quantity is modified by the presence of the new $U(1)$ chiral order parameter: we have found that $\chi^{\prime}$ (in the chiral limit $\sup \left(m_{i}\right) \rightarrow 0$ ) acts as an order parameter for the $U(1)$ axial symmetry above $T_{c h}$. This prediction of our model could be tested in the near-future Monte Carlo simulations on the lattice (but see also Ref. 32]).

Finally, in Section 6, we have found that the existence of the new $U(1)$ chiral condensate can be directly investigated by studying (at $T=0$ ) the radiative decays of the pseudoscalar mesons $\eta$ and $\eta^{\prime}$ in two photons. A first comparison of our results with the experimental data has been performed at the end of Section 6: the results are encouraging, pointing towards a certain evidence of a non-zero $U(1)$ axial condensate (i.e., $F_{X} \neq 0$ ). However, one should keep in mind that our results have been derived from a very simplified model, obtained doing a first-order expansion in $1 / N_{c}$ and in the quark masses. We expect that such a model can furnish only qualitative or, at most, "semi-quantitative" predictions. Higher-order terms in $1 / N_{c}$ could give rise to corrections (in the "real world" with $N_{c}=3$ ) of the same order of magnitude of (or even larger than!) those induced by the new $U(1)$ axial condensate (having the form $\sim F_{X}^{2} / F_{\pi}^{2}$ ). Moreover, when going beyond the leading order in $1 / N_{c}$, it becomes necessary to take into account questions of renormalization-group behaviour of the various quantities and operators involved in our theoretical analysis. This issue has been widely discussed in the literature, both in relation to the analysis of $\chi_{c h}^{\prime}$, in the context of the proton-spin crisis problem [31], and also in relation to the study of the $\eta^{\prime}(\eta)$ radiative decays 38. Further studies are therefore necessary in order to continue this analysis from a more quantitative point of view.

Last, but not least, it would be also very interesting (for a comparison with future heavyion experiments) to extend our present analysis of the radiative decays to the non-zerotemperature case. We expect that some progress will be done along this line in the near future. 


\section{References}

[1] S.B. Treiman, in S.B. Treiman, R. Jackiw and D.J. Gross "Lectures in Current Algebra and Its Applications" (Princeton University Press, Princeton, 1972);

S.L. Adler and R.F. Dashen, "Current Algebras" (Benjamin, New York, 1968);

S. Coleman, Erice Lectures 1973, in "Laws of hadronic matter", Academic Press, London and New York (1975); reprinted in: S. Coleman, "Aspects of symmetry", Cambridge Univesity Press (1985).

[2] T. Blum, L. Karkkäinen, D. Toussaint and S. Gottlieb, Phys. Rev. D 51 (1995) 5153.

[3] S. Weinberg, Phys. Rev. D 11 (1975) 3583.

[4] G. 'tHooft, Phys. Rev. Lett. 37 (1976) 8;

G. 'tHooft, Phys. Rev. D 14 (1976) 3432.

[5] E. Witten, Nucl. Phys. B 156 (1979) 269.

[6] G. Veneziano, Nucl. Phys. B 159 (1979) 213.

[7] E. Meggiolaro, Phys. Rev. D 58 (1998) 085002.

[8] M. Teper, Phys. Lett. B 171 (1986) 81.

[9] A. Di Giacomo, E. Meggiolaro, H. Panagopoulos, Phys. Lett. B 277 (1992) 491.

[10] E.-M. Ilgenfritz, E. Meggiolaro and M. Müller-Preußker, Nucl. Phys. B (Proc. Suppl.) 42 (1995) 496.

[11] B. Allés, M. D’Elia and A. Di Giacomo, Nucl. Phys. B 494 (1997) 281.

[12] G. Veneziano, Phys. Lett. B 95 (1980) 90.

[13] E. Shuryak, Comments Nucl. Part. Phys. 21 (1994) 235.

[14] C. Bernard et al., Nucl. Phys. B (Proc. Suppl.) 53 (1997) 442;

C. Bernard et al., Phys. Rev. Lett. 78 (1997) 598.

[15] F. Karsch, Nucl. Phys. B (Proc. Suppl.) 83-84 (2000) 14.

[16] P.M. Vranas, Nucl. Phys. B (Proc. Suppl.) 83-84 (2000) 414. 
[17] E. Meggiolaro, Z. Phys. C 62 (1994) 669.

[18] E. Meggiolaro, Z. Phys. C 62 (1994) 679.

[19] E. Meggiolaro, Z. Phys. C 64 (1994) 323.

[20] E. Meggiolaro, "Remarks on the $U(1)$ axial symmetry in QCD at zero and non-zero temperature", preprint IFUP-TH/2002-24; hep-ph/0206236.

[21] E. Meggiolaro, "The U(1) axial symmetry and the chiral transition in $Q C D$ ", published in "Quark-Gluon Plasma and Heavy Ion Collisions", editors: W.M. Alberico, M. Nardi and M.-P. Lombardo (World Scientific, Singapore, 2002).

[22] R.D. Pisarski, F. Wilczek, Phys. Rev. D 29 (1984) 338.

[23] D. Kharzeev, R.D. Pisarski and M.H.G. Tytgat, Phys. Rev. Lett. 81 (1998) 512.

[24] A. Di Giacomo and E. Meggiolaro, Nucl. Phys. B (Proc. Suppl.) 42 (1995) 478.

[25] P. Di Vecchia and G. Veneziano, Nucl. Phys. B 171 (1980) 253.

[26] E. Witten, Annals of Physics 128 (1980) 363.

[27] C. Rosenzweig, J. Schechter and C.G. Trahern, Phys. Rev. D 21 (1980) 3388.

[28] P. Nath and R. Arnowitt, Phys. Rev. D 23 (1981) 473.

[29] K. Kawarabayashi and N. Ohta, Nucl. Phys. B 175 (1980) 477.

[30] M. Gell-Mann, R.J. Oakes and B. Renner, Phys. Rev. 175 (1968) 2195.

[31] G.M. Shore and G. Veneziano, Phys. Lett. B 244 (1990) 75;

G.M. Shore and G. Veneziano, Nucl. Phys. B 381 (1992) 23;

S. Narison, G.M. Shore and G. Veneziano, Nucl. Phys. B 433 (1995) 209;

S. Narison, G.M. Shore and G. Veneziano, Nucl. Phys. B 546 (1999) 235.

[32] L. Giusti, G.C. Rossi, M. Testa and G. Veneziano, Nucl. Phys. B 628 (2002) 234.

[33] G. Briganti, A. Di Giacomo and H. Panagopoulos, Phys. Lett. B 253 (1991) 427.

[34] A. Di Giacomo, E. Meggiolaro and H. Panagopoulos, Phys. Lett. B 291 (1992) 147. 
[35] P. Di Vecchia, F. Nicodemi, R. Pettorino and G. Veneziano, Nucl. Phys. B 181 (1981) 318.

[36] M. Teper, Phys. Lett. B 202 (1988) 553.

[37] M. Campostrini, A. Di Giacomo, Y. Günduc, M.P. Lombardo, H. Panagopoulos and R. Tripiccione, Phys. Lett. B 252 (1990) 436.

[38] G.M. Shore and G. Veneziano, Nucl. Phys. B 381 (1992) 3;

G.M. Shore, Nucl. Phys. B (Proc. Suppl.) 86 (2000) 368;

G.M. Shore, Nucl. Phys. B 569 (2000) 107;

G.M. Shore, Phys. Scripta T 99 (2002) 84. 